\title{
Study of a mini-array for the Linsley effect in cosmic-ray air showers
}

\author{
W E Hazen $\dagger$, H Y Dait and E S Hazen§ \\ $\dagger$ Department of Physics, University of Michigan, Ann Arbor MI 48109, USA \\ $\ddagger$ Institute of High Energy Physics, Academia Sinica, Beijing, People’s Republic of China \\ $\S$ Department of Physics, Boston University, Boston MA, USA
}

Received 25 April 1988, in final form 23 August 1988

\begin{abstract}
The arrival-time distribution of shower particles far from the core has been measured as a function of shower axis distance at the Akeno Observatory by a cluster of 64 scintillators (total area $16 \mathrm{~m}^{2}$ ) located near the centre of the $1 \mathrm{~km}^{2}$ array. More than 3500 $1 \mathrm{~km}^{2}$ array events and about $30020 \mathrm{~km}^{2}$ array events have been analysed. We compare parameters $\tau$ (exponential fit to arrival times), $\sigma$ (conventional dispersion) and $t_{\mathrm{r}}$ (rise time) for the specification of the spread in arrival times. From a measurement of time spread, the shower axis distance can be determined with an uncertainty of 25 to $30 \%$ with this detector system. The resulting uncertainty in shower size, including a $60 \%$ uncertainty in particle density measurement, is 120 to $140 \%$. The resultant smearing of changes in slope of the frequency spectrum of incident showers is calculated. The smearing masks all but the gross changes in slope. The method may be useful for anisotropy studies, when a system for finding direction is included.
\end{abstract}

\section{Introduction}

The study of high-energy physics and astrophysics by observation of cosmic rays is limited by the scarcity of the large-air-shower detector systems required for obtaining data. Mini-arrays of detectors that utilise the Linsley effect [1] may provide a simpler method, albeit more limited in its capability. The Linsley effect is the increase in spread of arrival times in a particle sample from a given shower with increasing distance from the shower centre. Thus, the measured time spread of particles striking a localised detector system can give the distance $(r)$ to the shower axis. The number of particles gives a measure of the local particle density $(\rho)$. The shower size $(N)$ can then be calculated from the lateral distributions of particles deduced by groups with conventional large arrays of detectors [2].

This experiment is a calibration of the potential of a $16 \mathrm{~m}^{2}$ cluster of 64 scintillators as a large-shower detector, utilising the Linsley effect [3]. Previous observations of the Linsley effect [2] have been largely confined to measurements of the effect itself, with only a little quantitative appraisal of the potential for large-shower studies. In the work reported here, we have (i) compared various methods of measuring the observed time spreads, including our new method of an exponential fit to the arrivaltime distribution for a given shower; (ii) determined the uncertainty in the shower size $(N)$ calculated from our scintillator cluster data alone; and (iii) appraised the effect of 


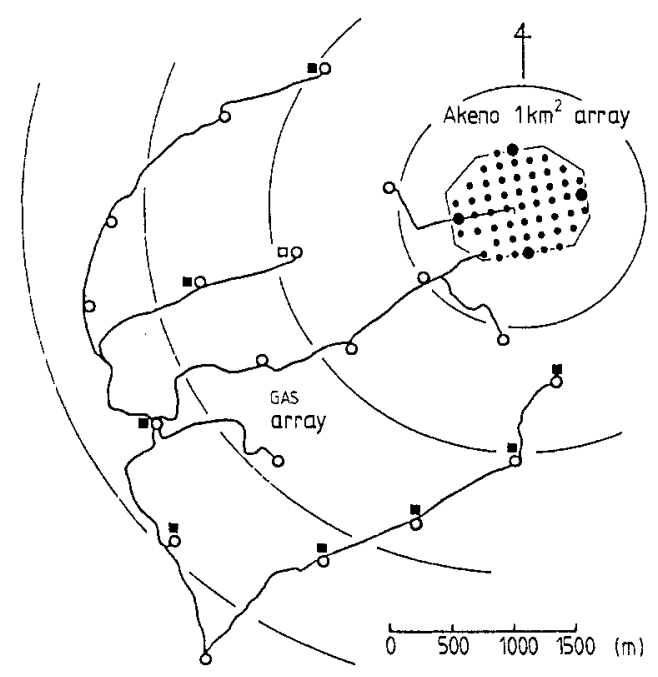

Figure 1. Detector arrangement of the Akeno array. The open and closed circles designate scintillation detectors of area $2.25 \mathrm{~m}^{2}$ and $1 \mathrm{~m}^{2}$ respectively. The irregular octagon outlines the $1 \mathrm{~km}^{2}$ array. The larger circles designate the GAs array.

the above uncertainty on the usefulness of a shower size spectrum obtained with our scintillator cluster.

\section{Experiment}

The experiment was performed at the Akeno Observatory of the Institute for Cosmic Ray Research of the University of Tokyo. We operated a cluster of 64 scintillators located on the roof of the central laboratory building, which is at the centre of the $1 \mathrm{~km}^{2}$ Akeno array of $531 \mathrm{~m}^{2}$ scintillators. The $1 \mathrm{~km}^{2}$ array is at the edge of the Akeno $20 \mathrm{~km}^{2}$ array (figure 1).

The cluster scintillators were standard Akeno detectors of $50 \mathrm{~cm} \times 50 \mathrm{~cm} \times 5 \mathrm{~cm}$ with only canvas and $\approx 1 \mathrm{~mm}$ of aluminium above the scintillator. The cluster configuration was eight close-packed rows of eight detectors with $50 \mathrm{~cm}$ spaces between rows. The detectors were operated as efficient single-particle (or more) detectors by setting the discriminators at the minimum of the free-run pulse-height spectrum. Then the efficiency was $\approx 95 \%$ and the noise $\approx 6 \%$.

In order to measure arrival times, the discriminator output of each detector was shaped to $25 \mathrm{~ns}$ then sent to a $40 \mathrm{~ns}$ resolution TDC. The TDCs are actually 64-bit shift registers with a shift rate of $25 \mathrm{MHz}$. The tDcs scanned approximately $2 \mu \mathrm{s}$. A further interval, from $\approx 2 \mu \mathrm{s}$ to $4 \mu \mathrm{s}$, was scanned by a $10 \mathrm{~ns}$ resolution TDC that received the fanned-in outputs of all 64 detectors. The TDC outputs were fed to an online microcomputer (Radio Shack TRS 80 Color Computer).

\subsection{Trigger}

The purpose of the mini-array is to scan the largest possible area consistent with a given uncertainty. Therefore we need to work with showers whose centres can fall up to a maximum distance determined by the minimum detected particle number that 
gives a tolerably small uncertainty. This number is of order $8-10$ for an uncertainty in shower size of the order of 30 per cent or so. Thus we are dealing with events where it is a good approximation that multiparticle hits on a detector are unlikely, i.e. the detectors are effectively single-particle counters.

A hardware trigger requiring only (a) a particle minimum in the range of 5-10 particles in our $16 \mathrm{~m}^{2}$ would give a rate of several hertz, which our recording system could not handle. Therefore, we added a requirement (b) for a minimum in the time spread, which, in effect, reduces triggers by showers with centres striking near our cluster. The result is to virtually eliminate triggers by small showers that must strike close enough to meet the minimum particle number requirement (a). Such showers consistute the main contribution to the rate of a trigger with only a particle number requirement (a). Triggers from close hits by large showers are also likely to be vetoed by (b), but the fraction of potential area lost thereby becomes small for large showers.

We used two parallel trigger circuits: (1) required four or more particles within the first $200 \mathrm{~ns}$ followed by at least two in a 200-2000 ns window, (2) required only two or more in the first $300 \mathrm{~ns}$ followed by two or more in a 300-2000 ns window. Either trigger (via the computer) activated the read-out and tape storage of the data. Trigger (1) also triggered storage of data of the Akeno $1 \mathrm{~km}^{2}$ array. The rate was $\approx 5 \mathrm{~h}^{-1}$. Trigger (2), with a rate of $\approx 1.8 \mathrm{~h}^{-1}$ was designed to be as unrestrictive as conveniently possible for more distant, very large showers.

\subsection{Collection of data}

As indicated above, our online computer stored the read-out of relative arrival times of the particles that struck the 64 cluster detectors. In addition, the arrival time of the shower was recorded from the read-out of our onboard clock. This clock was driven by signals from the AC power line. The clock was synchronised with the Akeno $20 \mathrm{~km}^{2}$ array clock to $\approx \pm 1 \mathrm{~s}$. The primary purpose of the clock was correlation with Akeno $20 \mathrm{~km}^{2}$ events, since there was no convenient way of arranging a hardware link.

The data from the Akeno $1 \mathrm{~km}^{2}$ array were taken from their tapes. In the case of the Akeno $20 \mathrm{~km}^{2}$ array, we used only their calculated values of shower parameters.

\subsection{Reduction of data}

The particle arrival-time data required only the shift register frequency for conversion to conventional time units.

The Akeno $1 \mathrm{~km}^{2}$ data were reduced to particle densities by using Akeno calibration data. A standard method was then used to calculate shower parameters for showers that hit within the array. These showers gave us information out to distances of $\approx 500 \mathrm{~m}$ from our detector cluster. For information out to $\approx 1000 \mathrm{~m}$, the shower parameters were obtained by seeking the azimuth of maximum density gradient to determine the direction to the shower axis outside the arrays, and then, using the density gradient to find the axis distance.

The Akeno $20 \mathrm{~km}^{2}$ (GAS) shower parameters were obtained from the analysis by the Akeno group [2].

\section{Results}

The running time was about one year, from which $\approx 35001 \mathrm{~km}^{2}$ events and $\approx 300$ $20 \mathrm{~km}^{2}$ (GAS) events were correlated for analysis. 


\subsection{Arrival-time distribution}

In advancing his idea of utilising shower disc thickness as another parameter for shower studies, Linsley [1] used the standard dispersion constant $\sigma$ as a measure of disc thickness. He was constrained to the use of $\sigma$ because his data were taken in the days of slow circuits, which manifested disc thickness only as a broadening of the pulses with distance from the shower axis. He also observed occasional resolved pulses that followed the main pulse and attributed them to something other than multiple scattering in the EM component. This implied that there would be occasional admixtures of these pulses with the EM component pulses, with consequent spurious increases in $\sigma$ for such events. This might occur in $\geqslant 15 \%$ of showers, as noted by Kakimoto et al [4]. Later studies [4], with circuits of higher time resolution, have made arbitrary cut-offs for 'late' pulses in calculating $\sigma$, and have also experimented with rise time $\left(t_{\mathrm{r}}\right)$ as a parameter, either of which reduces the problem.

We introduce another method for reducing the effect of delayed particles that are not part of the EM component: fitting the integral of each arrival-time distribution with an exponential. The resulting fit should be insensitive to a late particle or two.

We define the time distribution parameters as follows.

(a) Time dispersion $\sigma$, which is calculated according to $\sigma^{2}=\int p(t)\left(t_{i}-t\right)^{2} \mathrm{~d} t$, where $p(t)$ represents the differential arrival-time distribution and $t$ is the average arrival time. In our case of discrete times, it is defined as $\sigma^{2}=\sum_{i=1}^{n}\left(t_{i}-t\right)^{2} /(n-1)$, where $n$ is the number of particles.

(b) Rise time $t_{\mathrm{r}}$, as suggested by Kakimoto et al [4], is defined as the time between reaching $20 \%$ and $70 \%$ of the integral arrival-time distribution.

(c) Exponential constant $\tau$. In this case, we fit the integral arrival-time distribution by an exponential function $F(t)=1-\mathrm{e}^{-t / \tau}$. Figure 2 shows some illustrative examples. This parameter has the potential advantage of less sensitivity to late particles, as mentioned above.

The results of our measurements for arrival-time distribution as a function of shower-axis distance are shown in figure 3. The captions and labels show the nature of the sample. At distances less than a few hundered metres the trigger bias against nearby showers results in a bias in favour of the odd nearby shower with an unusually large time scatter. The effect is evident in the GAS results (figure 4). This bias is inconsequential since our interest is in greater distances.

It can be seen that our results for $\sigma$ are consistent with Linsley's results [1] within the uncertainty of our measurements, even though a best fit to our data would have a smaller slope. The point near $2000 \mathrm{~m}$ in the GAS results is based on only two events; consequently the short error bar is not truly representative.

\subsection{Deduction of axis distance}

The purpose of the Linsley method is to deduce the axis distance from the time spread measured by a localised detector. We will use the GAS results since they extend beyond one kilometre. The key question is the uncertainty in distance when determined from the time spread measurement. If we look at our parameter $\tau$, it is seen that the scatter in $\tau$ for a given $r$ is not very dependent on $r$ and averages $35 \%$ to $40 \%$ from 400 to $2000 \mathrm{~m}$. The contribution of uncertainty in $r$ to the above scatter in $\tau$ is probably not large enough to justify reduction of the above figure of $40 \%$. This 
assumption is based on the estimate by the Akeno group $[2,4]$ that the uncertainty in core location for GAS showers is about $50 \mathrm{~m}$ and in most cases less than $100 \mathrm{~m}$.

The time spread in the $400 \mathrm{~m}$ to $2000 \mathrm{~m}$ range can be expressed as

$$
\sigma \quad \text { or } \quad \tau \approx r^{-n}
$$

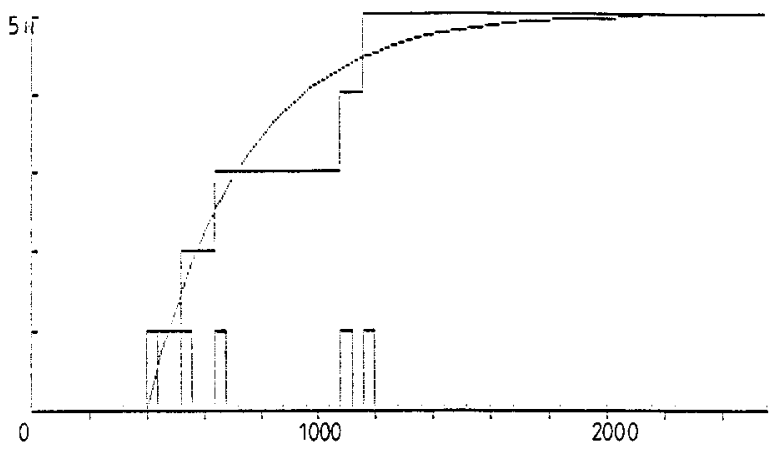

(a)

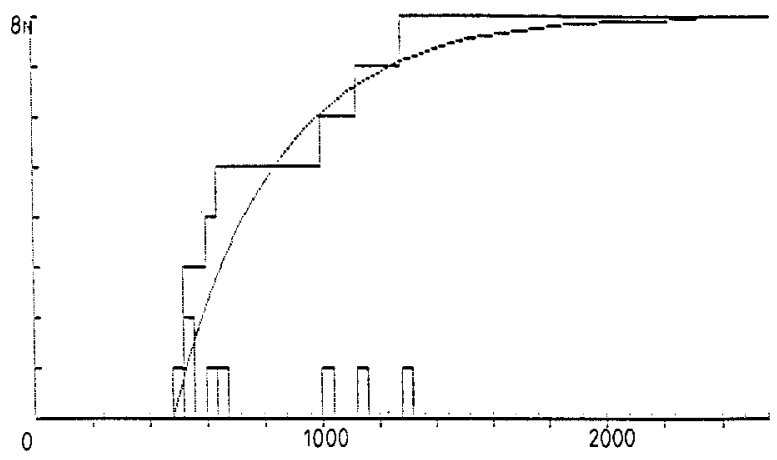

(b)

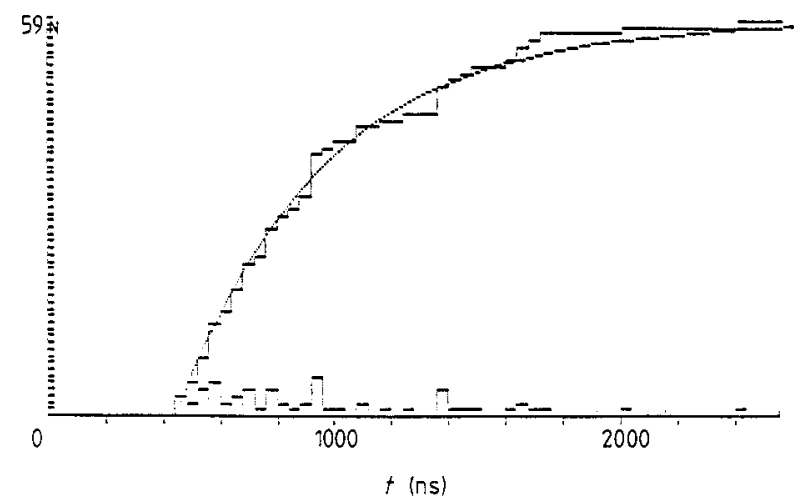

(c)

Figure 2. Examples of the arrival-time distributions. The curves represent exponential fits to the distributions (see text). (a) Run 37, event 410: $\tau=347 \mathrm{ns,} \mathrm{GAs} \mathrm{distance} 1136 \mathrm{~m}$. (b) Run 45, event 189: $\tau=367$ ns, Gas distance 1085. (c) Run 37, event 796: $\tau=521 \mathrm{ns,} \mathrm{GaS}$ distance $1229 \mathrm{~m}$. 

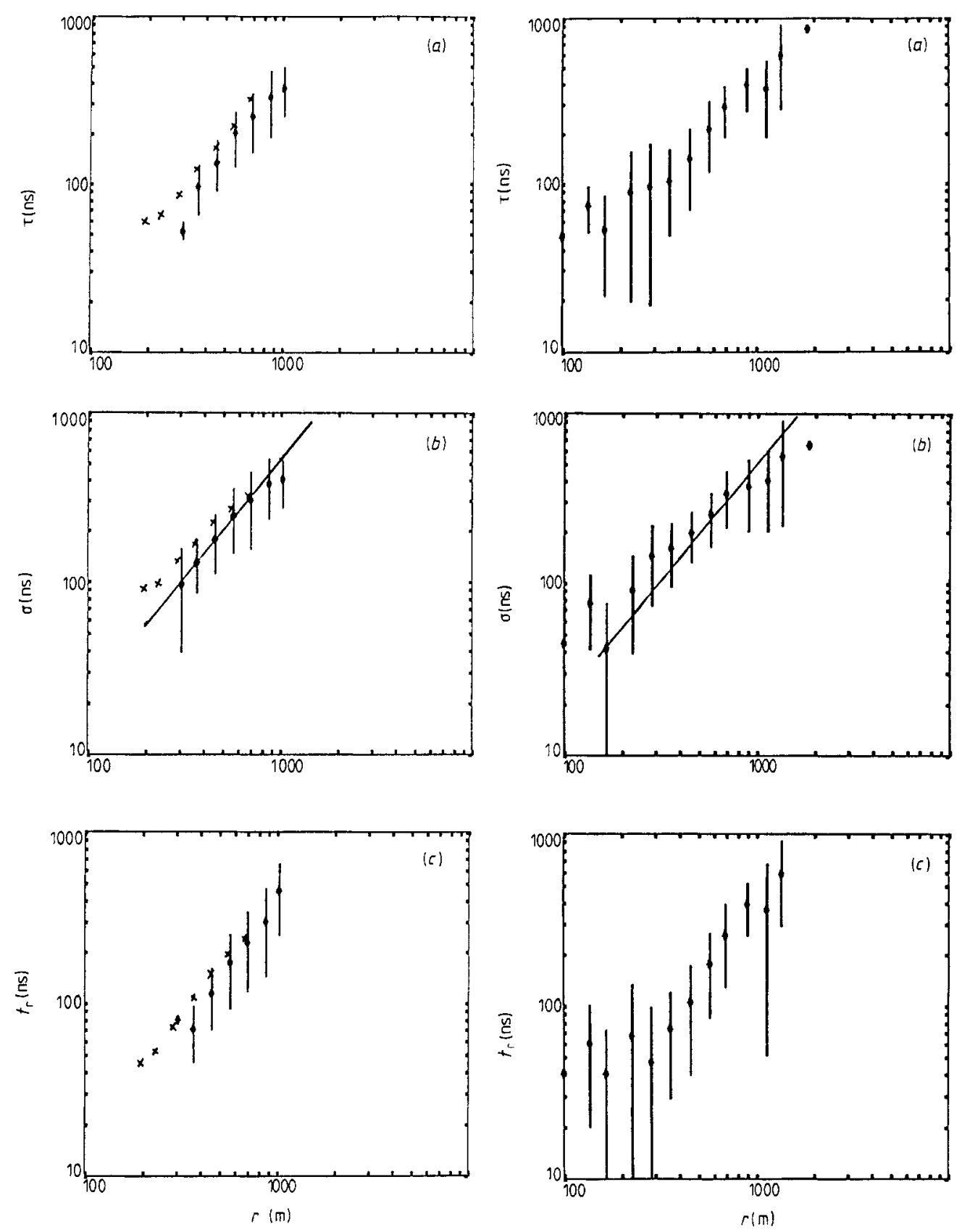

Figure 3. Time spread as a function of distance from the shower axis, with distance determined from the $1 \mathrm{~km}^{2}$ array data. The crosses represent results for smaller showers $\left(N \approx 10^{7}\right.$ to $\left.10^{8}\right)$ where trigger bias is seen at smaller distances, as expected. The principal data points (with associated error bars) are for shower size $>10^{8}$ and $\mathrm{ZA}<25^{\circ}$. The straight line in (b) plots Linsley's result [1].

Figure 4. Time spread as a function of distance from the shower axis, with distance determined from the GAS array. Data points for shower size $>10^{7.5}$ and $\mathrm{zA}<40^{\circ}$. The straight line in $(b)$ plots the Linsley result [1]. 
with $n$ about 1.4 to 1.5 for showers near the vertical. The uncertainty in distance resulting from a scatter of 35 to $40 \%$ in $\sigma$ or $\tau$ is, therefore, about 25 to $30 \%$.

\section{Mini-array as shower detector}

\subsection{Shower size}

Since the measured lateral density distribution of particles is only weakly dependent on shower size, $N$, [4], the size of a shower can be deduced from measurements of local particle density, $\rho$, and arrival time spread without much error without reiterations. In the range of $500 \mathrm{~m}$ to $2000 \mathrm{~m}$, the lateral density distribution is well represented by [4]

$$
\rho=1780 \mathrm{Nr}^{-4} \text {. }
$$

The uncertainty in shower size resulting from uncertainty in $r$ with our mini-array $(\$ 3.2)$ is, therefore, about 100 to $120 \%$.

The additional uncertainty due to scatter in the measurement of $\rho$ can be estimated from the Akeno studies of Teshima et al $[2,4]$, which give about $60 \%$ for our minimum of eight particles in $16 \mathrm{~m}^{2}$ of detector.

Thus, our estimate of overall uncertainty in shower size determined by our method is about 120 to $140 \%$.

\subsection{Size spectrum}

The uncertainty in shower size discussed above leads to a smoothing effect and potential masking of interesting features of the frequency spectrum of shower sizes incident on our type of mini-array. We have evaluated this effect by finding the result of standard error $\delta$ (in logarithmic scale) on an assumed incident spectrum.

The analysis is performed for an energy spectrum in place of a shower-size spectrum, since the common ground for discussion by shower study groups is the energy spectrum. The two are not strictly equivalent, but the departure from linearity is not sufficient to make a serious difference in the conclusions. As an example, an energy spectrum has been assumed as shown in figure 5 by the line with sharp changes in slope. Sharp changes are convenient for analysis and permit a clear picture of the smoothing effect. The assumed spectrum is close to the majority of observational data.

The effect of a standard error in energy determination, $\delta$, with an index $\gamma$ for a differential power spectrum is an increase of apparent flux by a factor $\exp \left[\frac{1}{2}(\gamma-1)^{2} \delta^{2}\right]$.

The results of this increase are shown in figure 5 for various values of $\delta$. The assumed slope changes are marginally detectable, even for the value $\delta=1$ that is about the error found in $\$ 4.1$.

\section{Discussion and conclusions}

Our version of a mini-array appears to be only marginally useful for the study of the high-energy end of the frequency spectrum because of the attenuation of the possible changes in slope. The expected rate of collection of data would be tolerable, namely, about five showers per year under our condition of $\rho$ minimum of eight particles in $16 \mathrm{~m}^{2}$, with $E \geqslant 10^{19} \mathrm{eV}$. 


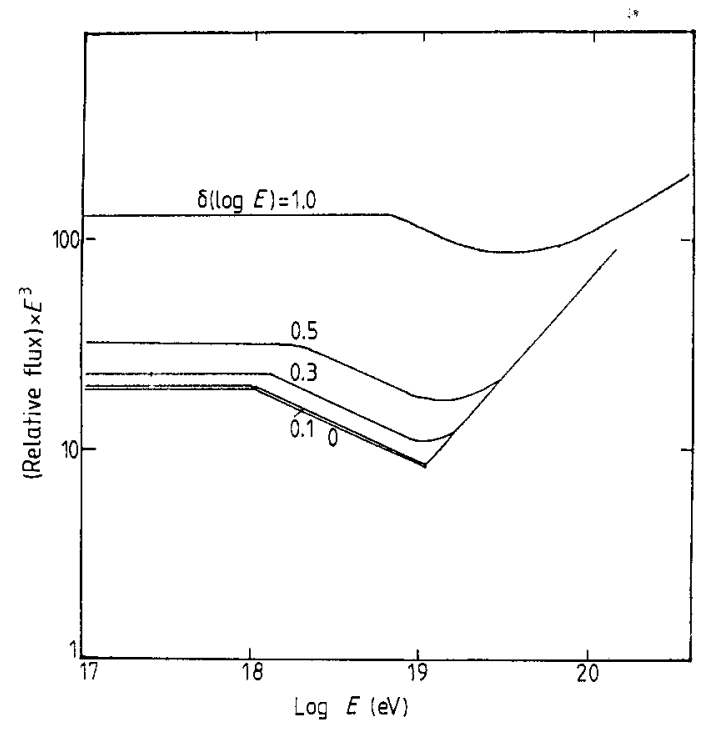

Figure 5. The modulation of an assumed energy spectrum by an uncertainty $\delta(\log E)$ in the measurement of $E$. The power-law slopes of the assumed spectrum 3, 3.36, and 2.1 give a simplified version of a reasonable representation of world results (e.g. Linsley $J$ 1985 Proc. 19th ICRC, LaJolla 9 475).

It looks more promising for studies of anisotropy at high energies, where the uncertainty in energy is less likely to mask the interesting phenomena. However, it would be necessary to change to a detector system such as two layers of streamer tubes in order to determine local particle directions in addition to local density and time spread. This would probably be adequate for studies of gross anisotropy, but probably not adequate for point-source searches.

\section{Acknowledgments}

Our experiment was possible only because of the enthusiastic support given to us by all members of the ICRR staff at Akeno and Tanashi. Financial support from the USA was provided by the NSF and the University of Michigan.

\section{References}

[1] Linsiey J 1986 J. Phys. G: Nucl. Phys. 1251

[2] Teshima M et al 1986 J. Phys. G: Nucl. Phys. 121097

[3] Hazen W E et al 1987 Proc. 20th ICRC Moscow HE3.3-12

[4] Kakimoto F et al 1985 Proc. 19th ICRC, La Jolla 7328

Lawrence M A et al 1985 Proc. 19th ICRC, La Jolla 7332

Linsley J 1985 Proc. 19th ICRC, La Jolla 7359

See also Teshima et al [2] 